Recepción: 15 / 04 / 2017

Ciencias Médica

Aceptación: 01 / 05 / 2017

Articulo Científico

Publicación: 15 / 05 / 2017

\title{
Estudio de la ficha dental en la Facultad Piloto de Odontología durante el periodo 2013
}

\author{
Study of the dental record in the Faculty Pilot of Dentistry during the \\ period 2013
}

\section{Estudo da arcada dentária na Escola Piloto de Odontologia para o período de 2013}

\author{
Carlos G. Martínez-Florencia \\ carlos.martinezf@ug.edu.ec
}
María E. Benítez-Hoyos carcemil@hotmail.com

José M. González-Benavides "' jose.gonzalezb@ug.edu.ec

Correspondencia: carlos.martinezf@ug.edu.ec

\footnotetext{
Magister en Medicina Forense; Doctor en Odontología; Universidad de Guayaquil, Ecuador.

Magister en Salud Pública; Doctora en Odontología; Universidad de Guayaquil, Ecuador.

Magister en Diseño Curricular; Licenciado en Sistemas de Información; Odontólogo; Universidad de Guayaquil, Ecuador.
} 


\section{Resumen}

La presente investigación tiene como propuesta implementar un método de identificación odontografica en medicina forense. Esto se justifica debido a la incertidumbre respecto a la magnitud de los daños sufridos y a la falta de información para identificar a seres humanos víctimas de desastres, vale resaltar la dificultad de aplicar medidas de emergencia inmediatas. Sin embargo, es necesario establecer urgentemente la coordinación a todos los niveles (local, regional, nacional. Aunque los planes de respuesta a catástrofes suelen prever mecanismos de coordinación, es posible que no se pongan en marcha inmediatamente después de la catástrofe y por lo general no están concebidos para el caso en cuestión. Al poner en práctica las primeras medidas de emergencia, es muy importante ocuparse con eficacia y a la mayor brevedad de la identificación de cadáveres. También hay que considerar que el único alivio para los familiares de un desaparecido reside en la confirmación creíble de su muerte y en el conocimiento de que los restos han sido o serán tratados con dignidad, Cada caso debería ser cuidadosamente considerado antes de emitir una conclusión de identificación para la presente desarrollo se consideraron: En la presente investigación se realizó un estudio descriptivo, transversal a través de encuestas a docente y estudiantes del último año de la carrera odontología de la Universidad de Guayaquil y médicos forenses. Orientado a determinar el diagnóstico acerca del nivel de conocimiento que tienen sobre el proceso de identificación humana en casos de desastres.

Palabras clave: Ficha dental; método de identificación; odontografica; medicina forense. 


\begin{abstract}
The present research is proposed to implement a method odontografica identification in forensics. This is justified because of the uncertainty regarding the extent of damage and the lack of information to identify disaster victims humans, it highlight the difficulty of applying immediate emergency measures. However, it is necessary to urgently establish coordination at all levels (local, regional, national. Although disaster response plans typically provide coordination mechanisms, may not be put in place immediately after the disaster and usually are not designed for the case. in implementing the first emergency measures, it is very important to deal effectively and promptly identifying corpses. also consider that the only relief for relatives of a missing resident on credible confirmation of his death and the knowledge that the remains have been or will be treated with dignity, each case should be carefully considered before a conclusion of identification for this development were considered: in the present investigation was conducted a descriptive, crosssectional study using surveys to teachers and senior students of dentistry career at the University of Guayaquil and coroners. Aimed at determining the diagnosis about the level of knowledge they have about the process of human identification disaster.
\end{abstract}

Key words: Dental record; identification method; odontografica; forensic medicine. 


\section{Resumo}

A presente pesquisa é proposta para implementar um método odontografica identificação em forense. Isso se justifica por causa da incerteza quanto à extensão dos danos e à falta de informações para identificar as vítimas de desastres humanos, destaca a dificuldade de aplicar medidas imediatas de emergência. No entanto, é necessário estabelecer urgentemente uma coordenação a todos os níveis (local, regional, nacional). Embora os planos de resposta a catástrofes normalmente forneçam mecanismos de coordenação, não podem ser postos em prática imediatamente após a catástrofe e normalmente não são concebidos para o caso. Considera que o único alívio para os parentes de um desaparecido residente sobre a confirmação credível da sua morte e o conhecimento de que os restos mortais foram ou serão tratados com dignidade, cada um deles Deve ser cuidadosamente considerada antes de se considerar uma conclusão de identificação para este desenvolvimento: no presente estudo foi realizado um estudo descritivo, transversal, utilizando-se inquéritos a professores e estudantes seniores de carreira odontológica da Universidade de Guayaquil e coroners. O diagnóstico sobre o nível de conhecimento que têm sobre o processo de identificação humana Desastre.

Palavras chave: Registro dental; método de identificação; odontografica; forense. 


\section{Introducción.}

Es ampliamente conocida la utilidad de la evidencia dental y la importancia de la historia clínica para la identificación humana en una variedad de situaciones médico legales. Aún después de muchos años de la muerte, los dientes son los órganos mejor preservados y los más valiosos indicadores de la identidad de las personas.

El uso de la información dental para identificar personas desconocidas es una parte esencial de la respuesta a desastres masivos, los cuales cada día son más comunes. La confección de buenas historias clínicas no es suficiente para que los datos recogidos en estas puedan ser utilizados con fines identificativos; se hace indispensable la existencia de una ficha dental con su correspondiente sistema de archivo. (Miranda J, 1994.)

El tratamiento dental por sí mismo es la mayor contribución a la unicidad de la dentición de un individuo, y junto con las características del desarrollo, es la llave para lograr la identificación humana. Aún después de muchos años de la muerte, los dientes son los órganos mejor preservados y los más valiosos indicadores de la identidad de las personas. El uso de la información dental para identificar personas desconocidas es una parte esencial de la respuesta a desastres masivos, que cada día son más comunes. (Vale GL, 2004)

El objetivo de la presente investigación es Analizar la Ficha Dental utilizada en la Facultad Piloto de Odontología durante el periodo 2013 de pacientes de mayores de 18 años y proponer un método que permita la identificación odontografica en Medicina Forense. 


\section{Materiales y métodos.}

\section{Tipo de investigación}

Según el problema y el objetivo, la presente investigación es un estudio de tipo transversal, descriptivo retrospectivo.

\section{Universo}

La muestra la constituyen las 20 fichas clínicas de la Facultad Piloto de odontología del periodo 2013.

\section{Criterios de inclusión}

Fichas dentales de mayores de 18 años

\section{Criterios de exclusión}

Fichas dentales de menores de 18 años

Encuestas a alumnos sobre la importancia del tema a investigar

\section{Factibilidad y recursos para la ejecución}

La presente propuesta es factible ya que se cuenta con el apoyo de las Autoridades de la Facultad Piloto de Odontología. Además los recursos económicos los asume el Autor de la propuesta de investigación. 


\section{Resultados.}

En la presente investigación se realizó un estudio descriptivo, transversal a través de encuestas a docente y estudiantes del último año de la carrera odontología de la Universidad de Guayaquil y médicos forenses. Orientado a determinar el diagnóstico acerca del nivel de conocimiento que tienen sobre el proceso de identificación humana en casos de desastres.

Se encuestaron a 80 estudiantes y 15 odontólogos y 3 especialistas en medicina forense. La encuesta estuvo orientada a determinar el nivel de conocimiento sobre la historia clínica en la identificación de cadáveres en desastres

\section{Análisis de resultados}

¿Qué tipo de cadáveres necesita ser identificado por medio de la Historia clínica?

\begin{tabular}{|c|c|c|}
\hline ALTERNATIVA & FRECUENCIA & PORCENTAJE (\%) \\
\hline Quemados & 98 & 100 \\
\hline Atropellados & 0 & 0 \\
\hline TOTAL & $\mathbf{9 8}$ & $\mathbf{1 0 0}$ \\
\hline
\end{tabular}

Cuadro $N^{0} 1 .-$ Encuestas

En el grafico $\mathrm{N}^{\mathrm{o}} 1$ se observa en relación a la interrogante: El tipo de cadáveres que necesita ser identificado por medio de la historia clínica: el $100 \%$ de los encuestados manifiesta que los quemados.

¿Usted conoce la técnica de la identificación de cadáveres por medio de los tratamientos odontológicos realizados? 
Carlos G. Martínez-Florencia; María E. Benítez-Hoyos; José M. González-Benavides

\begin{tabular}{|c|c|c|}
\hline ALTERNATIVA & FRECUENCIA & PORCENTAJE (\%) \\
\hline $\mathrm{Si}$ & 98 & 100 \\
$\mathrm{No}$ & 0 & 0 \\
\hline TOTAL & $\mathbf{9 8}$ & $\mathbf{1 0 0}$ \\
\hline
\end{tabular}

Cuadro $N^{o}$ 2.- Fuente encuestas

Se aprecia en relación a la pregunta: El conocimiento de la técnica de la identificación de cadáveres por medio de los tratamientos odontológicos realizados. El 100\% de los encuestados responden que si conocen y aplican la identificación por medio de tratamientos odontológicos

3. ¿En qué tipo de cadáveres existen limitaciones para la identificación?

\begin{tabular}{|c|c|c|}
\hline ALTERNATIVA & FRECUENCIA & $\begin{array}{c}\text { PORCENTAJE } \\
(\mathbf{\%})\end{array}$ \\
\hline Incinerados & 9 & 9,18 \\
\hline Ahogados & 46 & 46,94 \\
\hline Decapitados & 5 & 5,10 \\
\hline Choques & 17 & 17,35 \\
\hline Muertes violentas & 21 & 21,43 \\
\hline TOTAL & $\mathbf{9 8}$ & 100 \\
\hline
\end{tabular}

El cuadro $\mathrm{N}^{\mathrm{o}} 3$ se aprecia en relación a la interrogante: el $46.94 \%$ responden ahogados, el $21,43 \%$ muertes violentas, el 17,35\% x hoques, el 9,18\% incinerados y el 5,10\% decapitados. 
¿Usted piensa que es importante que en los departamentos de medicina legal, indispensable la historia clínica de pacientes que perdieron la vida en desastres?

\begin{tabular}{|c|c|c|}
\hline ALTERNATIVA & FRECUENCIA & $\begin{array}{c}\text { PORCENTAJE } \\
(\boldsymbol{\%})\end{array}$ \\
\hline $\mathrm{Si}$ & 69 & 70 \\
\hline $\mathrm{No}$ & 29 & 30 \\
\hline TOTAL & $\mathbf{9 8}$ & $\mathbf{1 0 0}$ \\
\hline
\end{tabular}

La importancia de que en los departamentos de medicina legal, indispensable la historia clínica de pacientes que perdieron la vida en desastres. Se aprecia en relación de la importancia del interrogante: el $70 \%$ el $30 \%$ no.

¿Cuáles son las limitaciones durante la autopsia por la ausencia de la historia clínica?

\begin{tabular}{|c|c|c|}
\hline ALTERNATIVA & FRECUENCIA & PORCENTAJE (\%) \\
\hline Ninguno & 23 & 23,47 \\
\hline Autopsia & 68 & 69,39 \\
\hline $\begin{array}{c}\text { Certificado } \\
\text { defunción }\end{array}$ & 7 & 7,14 \\
\hline TOTAL & $\mathbf{9 8}$ & $\mathbf{1 0 0}$ \\
\hline
\end{tabular}

La medicina legal que es la ciencia que tiene por objeto el estudio de cuestiones que se presentan el ejercicio profesional, las limitaciones durante la autopsia por la ausencia de la historia clínica: el $23.47 \%$ manifiestan que ninguno, en cambio el $69.39 \%$ prefieren la autopsia y no la historia clínica. 


\section{¿Usted ha participado en autopsias de cadáveres incinerados?}

\begin{tabular}{|c|c|c|}
\hline & & $\begin{array}{c}\text { PORCENTAJE } \\
\text { ALTERNATIVA }\end{array}$ \\
\hline $\mathrm{Si}$ & FRECUENCIA & 0 \\
\hline $\mathrm{No}$ & 0 & 100 \\
\hline TOTAL & $\mathbf{9 8}$ & $\mathbf{1 0 0}$ \\
\hline
\end{tabular}

Se deduce que los médicos legistas no han participado en autopsias de cadáveres incinerados

¿Cómo Forense cuales son las identificaciones de cadáveres que más se utilizan?

\begin{tabular}{|c|c|c|}
\hline ALTERNATIVA & FRECUENCIA & $\begin{array}{c}\text { PORCENTAJE } \\
(\boldsymbol{\%})\end{array}$ \\
\hline Por huella & 98 & 100 \\
\hline No contesta & 0 & 0 \\
\hline TOTAL & $\mathbf{0}$ & $\mathbf{0}$ \\
\hline
\end{tabular}

Las identificaciones de cadáveres la mayoría de los legistas encuestados lo hacen por huellas $100 \%$

\section{Discusión.}

La autopsia médico-legal o judicial constituye uno de los procedimientos de mayor relevancia entre los realizados por la actividad médico forense

Su importancia radica en el poder determinar la verdadera causa de la muerte: Natural o Violenta, y en este último caso, si fue Accidental, Suicida u Homicida.

El circunscribirla meramente a una tarea de identificación y cotejo de elementos dentarios es reducir la envergadura y potencial de su aporte a la faz forense. 
Por otra parte, es innegable que su actuación formando parte del procedimiento médico legal necesita de profesionales capacitados.

\section{Propuesta.}

\section{"Método de identificación odontografica en medicina forense"}

Historia clínica.- La función principal de la Historia Clínica es de orden asistencial. Es el medio básico que posibilita la correcta comunicación y la actuación eficaz de los miembros de los diferentes equipos asistenciales que puedan intervenir en el cuidado de cada uno de los pacientes.

\section{Características:}

Veracidad.- Registro real de las decisiones y acciones de los profesionales responsables sobre los problemas de la salud del usuario

Integralidad.- Información completa sobre las fases de promoción de la salud, prevención, diagnóstico, tratamiento y rehabilitación de la enfermedad del usuario

Pertinencia.- Aplicación de criterios de racionalidad científica en el registro de los datos, de conformidad con las normas y protocolos de atención.

Secuencialidad.-Mantenimiento de un orden cronológico de los formularios

Disponibilidad.- Existencia real y completa de los formularios y documentos complementarios para su utilización en el momento requerido.

Oportunidad.- Registro simultáneo de los datos mientras se realiza la atención Ministerio de Salud Pública del Ecuador, MANUAL DE 
Calidad del registro.- Llenado completo con claridad, legibilidad y estética, (evitando siglas o símbolos no autorizada) Uso de los formularios básicos de la historia clínica única, Quito Ecuador, 2008

Objetivo: Implementar un método de historia clínica con identificación odontografica para medicina forense.

Justificación de la propuesta.- La ficha o historia clínica odontológica es la herramienta que utiliza el odontólogo para el registro de la información dental. (Ciocca Gómez)

Ordinograma o dentigrama, norma esquemática que le permite la codificación de esa información, no solo en la práctica clínica sino también en la confección de fichas y sistemas específicos para la identificación forense. (INTERPOL)

Sin embargo, este recurso gráfico aún no cuenta con un consenso de lenguaje internacional y ha sido cuestionada su configuración por la imposibilidad de registrar datos potencialmente identificatorios. (Fonseca et al., 2010; Varkkola).

Se ha sugerido un mayor recaudo en la obtención y archivo de datos imagenológicos (radiografías, fotografías, modelos, etc.) pues permiten registrar información despojada de subjetividad y difícilmente codificable en un odontograma (malposiciones, pigmentaciones, lesiones, etc.). Esto posibilita asentar datos morfológicos incluso en ausencia de restauraciones (situación frecuente en niños y jóvenes) o edentulismo, y facilita la transmisión de información desligada de barreras lingüísticas. (Solheim et al., 1992; Andersen et al., 1995).

Quizás una de las mayores utilidades de codificar la información dental es la posibilidad de cuantificar los rasgos de cotejo y las observaciones. Aunque se ha coincidido en que la comparación 
de las informaciones AM-PM solo puede ofrecer resultados categóricos: identificación inequívoca, probable, posible, descartada o comparación imposible (INTERPOL), y que la utilización de este modelo cualitativo no implica falta de fiabilidad dada la complejidad de informaciones que deben compararse. (Dorion, 1990)

Martin de las Heras et al., 2010; Fonseca et al.) la cuantificación analítica del cotejo (tendencia acentuada con la entrada en escena de las pruebas genéticas) exige hoy significación estadística en las metodologías utilizadas. Steadman et al. (2007) en un interesante intercambio epistolar defienden que las comparaciones odontológicas y dactiloscópicas son útiles pero no dan certeza probabilística, pues el resultado categórico de un cotejo de informaciones AM-PM no puede ser corroborado de ninguna manera. (Anderson, 2007)

Los modelos matemáticos desarrollados en 1977 por Keiser-Nielsen y periódicamente reformulados para poblaciones específicas, continúan defendiendo a las trillonésimas posibles combinaciones de rasgos visibles en las 5 caras de los 32 dientes humanos (rangos similares a los de una perfilación genética) como herramienta de certeza para una identificación inequívoca (Martin de las Heras et al.). Sin embargo, los hechos han logrado rebatir este modelo teórico.

Especificaciones.- Indicar de quien es el cadáver que se ha encontrado

- Determinar si la causa de la muerte tiene como origen algún acto directivo.

- Conocer a la víctima y también al posible victimario

- Enrejar el cuerpo a los familiares

- Tramites de documento de identidad en personas abandonadas. 
Áreas de Trabajo.- En la identificación de cadáveres recientes, Registrando el dentigrama y características dentales post morten y realizando análisis comparativos con los datos proporcionados en la ficha premorten proporcionados por los familiares. Determinando la edad, Determinando la raza

Ficha Dental.- La ficha dental es un registro en los alumnos y profesionales de la salud oral describen la historia clínica del paciente. Actualmente debido al gran desarrollo que ha adquirido esta especialidad, ha obligado a que además de la ficha dental se adjunte e esta una historia clínica completa:

- Forma de las arcadas dentarias

- Numero presente de piezas dentarias

- Numero ausentes de piezas dentarias

- Restos radiculares

- Malposiciones dentarias

- Cavidades de caries

- Preparaciones y restauraciones

- Tratamientos protésicos. Endodonticos y ortodoncico

- Anomalías congénitas y o adquiridas

- Procesos infecciosos bucodentales presentes

Los métodos de la identificación odontológicas se fundamentan principalmente, en la particularidad de la conformación de las arcadas dentarias, dientes, rugosidades palatinas, que presentan cada individuo, con caracteres y formas propias. 
Odontometria.- Este método de identificación se basa en la obtención de medidas de uno o varios dientes que se hallan a disposición en el caso. Los trabajos más importantes de este método corresponden a Gysi, Bonwill, Hanley.

Aunque estos autores consideran a la Odontometría solo como ciencia básica al estudio especulativo de la ortodoncia para establecer la normalidad. Berttinni dice que la concepción matemática fundamental de la Odontometría más completa la débenos al argentino Dr. Carrera.

Rugopalatinoscopia.- Es el estudio de las impresiones de las rugosidades palatinas utilizadas para la identificación de personas. Rugopalatinoscopia procede las voces griegas "rughos" rugosidad y "Skopein" observaciones o examen. Al estampado del dibujo papilar y a su representación gráfica con fines didácticos o de estudio se denomina rugopalatinograma

Prostodoncia.- Es un método de identificación que se vale de ciertos signos convencionales que casi siempre prestan utilidad sobre todo cuando estos signos se graban en los aparatos protésicos. El Dr. Jeffejs fue el primero en Norteamérica en valerse de este sistema y el Dr. Echegaray y Corte en la Universidad de Antioquia, Medellín.

Este método como medio de identificación lo podemos aprovechar colocando en la prótesis el nombre del paciente, sexo. Edad, grupo sanguíneo y otros datos de importancia, este método lo emplean los odontólogos para identificar sus trabajos.

Odontograma.- Es un esquema de las arcadas dentarias, una cedula personal cuyo diagrama dental consigna las características anatómicas de los dientes, así como de las particularidades y modificaciones, con fines de identificación. Como elemento identificatorio está basado en un 
principio general, un mismo individuo no posee los dientes iguales, ni tampoco existen dos bocas iguales.

Odontoscopia.-Existen una serie de elementos que contribuyen de manera eficaz en los objetivos de la criminalística, entre ellos tenemos la Odontoscopia ósea las huellas o marcas de mordida hallazgos frecuentes en una serie de delitos

Queiloscopia.-Se refiere al estudio, registro y clasificación de los surcos de la mucosa externa de labio y de las huellas que deja

\section{Responsabilidades del medico forense}

Normalmente dos odontólogos cooperarán en el registro de la situación de los dientes del cadáver y en la elaboración de un expediente radiológico y fotográfico: uno de ellos será un odontólogo forense que realizará el examen y otro un odontólogo forense que se encargará del registro o un asistente de radiografía odontológica forense.

\section{Odontólogo forense encargado del examen}

El odontólogo encargado del examen accederá a la cavidad oral utilizando los procedimientos necesarios para, por ejemplo, practicar incisiones en los tejidos blandos (si procede), limpiar las piezas dentarias y los maxilares, examinar las estructuras y evaluar el estado de los dientes del cadáver.

Se realizarán radiografías de ambos lados de los molares con los dos maxilares unidos (aleta de mordida); de los molares superiores e inferiores y, si es posible, de los premolares e incisivos (periapicales); y de los dientes con características especiales, tales como endodoncias, coronas, etc.; 
también se hará cualquier otra radiografía que sea necesaria (oclusal, lateral oblicua de la mandíbula inferior c.)

Se evaluará la calidad de estas radiografías (exposición, densidad y nitidez) y después se estudiarán con el odontólogo encargado del registro (cuyas tareas se definen a continuación) para garantizar que se registren todos los datos en las páginas F1 y F2. El odontólogo encargado del examen también supervisará y dirigirá la elaboración de un expediente fotográfico apropiado de los dientes, maxilares, estructuras bucales relacionadas y rasgos y características dentales.

\section{Odontólogo forense encargado del registro}

El odontólogo encargado del registro ayudará al odontólogo encargado del examen a registrar el estado de los dientes de la víctima. El odontólogo encargado del registro preparará y completará las páginas F1 y F2, y registrará la información odontológica que le dicte el odontólogo encargado del examen; revisará el registro PM para garantizar su calidad (exactitud, legibilidad, claridad); firmará el registro y se asegurará de que el odontólogo encargado del examen también lo firme.

\section{Asistente de radiografía odontológica forense}

El asistente de radiografía odontológica ayudará a los odontólogos encargados del examen y del registro a preparar, tomar y revelar las radiografías de los dientes y compartirá la responsabilidad sobre la calidad de las radiografías PM. 


\section{Aspectos legales}

En 1967, se creó el Mi misterio de Salud Pública, hasta ese momento, los formularios de la Historia Clínica, tenían un formato diseñado de acuerdo a criterios clínicos definidos por los diferentes servicios de salud.

En 1972, mediante Decreto Supremo $\mathrm{N}^{\circ}$ 200, se estableció la obligatoriedad la Historia Clínica Única y del Sistema Estadístico, como un conjunto de formularios y métodos de registro médico y estadístico, a ser utilizados en los establecimientos hospitalarios del Estado, instituciones de derecho público, semipúblico y autónomas y en las instituciones de derecho privado, de acuerdo con el instructivo y manual de procedimientos dictado por el Ministerio de Salud Pública

Entre 1995 y 1996, la Dirección General de Salud y la Dirección de Planificación, integraron una comisión ministerial para analizar las posibilidades de actualización de la Historia Clínica y procede a elaborar la propuesta de rediseño de los formularios básicos para su presentación ante el Consejo Nacional de Salud

En agosto de 2005, el documento fue enviado al Ministerio de Salud Pública, al Proceso de Normalización quien analizó el documento y propuso un pilotaje de validación de la propuesta en la red de establecimientos del Ministerio de Salud Pública.

En octubre de 2006, el Directorio del Consejo Nacional de Salud, aprobó el expediente único para la Historia clínica, con 14 formularios básicos.

En enero del 2007, mediante Acuerdo Ministerial se aprobó la utilización de los formularios básicos actualizados de la Historia Clínica Única y de los formularios del "Registro Médico 
Orientado por Problemas" y se dispuso al Proceso de Aseguramiento de la Calidad la elaboración un plan de implementación evaluación de los formularios de la Historia Clínica, aprobados por el CONASA, (incluyendo 4 formularios adicionales)

En ese mismo año, el equipo técnico de la Comisión de Historia Clínica realizó la implementación evaluación de los formularios actualizados en 80 unidades operativas del MSP en las provincias de Guayas y Pichincha, cuyos resulta dos establecieron que el $72 \%$ de los participantes aprobó el documento sin cambios, mientras que el $28 \%$ presentó observaciones, principalmente al formato

Posteriormente, la Comisión Ministerial de la Historia Clínica, con apoyo técnico de OPS, consolidó los aportes recopilados en la fase de implementación evaluación y presentó 20 formularios básicos para su aprobación y aplicación

\section{Conclusiones.}

En base a los objetivos propuestos en la presente investigación concluimos:

Debido a su carácter único, las estructuras y rasgos de los dientes y las mandíbulas humanos son idóneos para la identificación de víctimas supervivientes o fallecidas.

Los datos dentales pueden recopilarse y registrarse en el momento del examen y compararse con datos aportados por médicos generalistas o dentistas que trataron a la víctima a lo largo de su vida.

Los dientes están protegidos en la cavidad bucal y soportan bien los efectos del ambiente exterior antes, durante y después de la muerte. 
Los dientes están hechos con las sustancias más duras y resistentes del cuerpo, de modo que mientras los tejidos blandos del organismo se deterioran, los rasgos dentales, tan valiosos para la identificación de víctimas, conservan su integridad.

Esta afirmación se aplica particularmente a los tratamientos dentales, como las obturaciones y coronas terapéuticas o estéticas, las desvitalizaciones y las prótesis dentales, ya que son tratamientos de encargo, únicos para cada persona.

\section{Bibliografía.}

Anderson, B. E. Statistical basis for positive identification in forensic anthropology. Am. J. Phys. Anthropol., 133(1):741; author reply 741-2, 2007.

Aparicio D, Henríquez L, Hurtado A, Pedraza A, Casas J. (2007). Identificación positiva por medio del uso de la rugoscopia en un municipio de Cundinamarca (Colombia). Acta Odontol Venez. 45(3):1-7

Alegría J, De Jesús C, Flores K, Godinez A, Peña E. (2008) Rugoscopia, una alternativa como medio de identificación de los alumnos de clínica molinito inscritos en el periodo -I - 2008-II

Boscolo- Berto R, Viel G, Cecchi R, Terranova C, Vogliardi S, Bajanowski T, Ferrara SD. (2012) Journals publishing bio-médico legal research in Europe. Int J Legal Med;126:129-37.

Brown, K. A. Procedures for the collection of dental records for person identification. J. Forensic Odontostomatol. 25(2):63-4, 2007.

Castilla J, Ramos V. (2004). Organización de la medicina legal en España. En: Villanueva E, editor. Gisbert

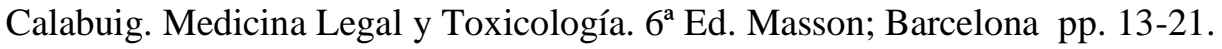

Cattaneo, C.; Porta, D.; De Angelis, D.; Gibelli, D.; Poppa, P. \& Grandi, M. Unidentified bodies and human remains: An Italian glimpse through a European problem. Forensic Sci. Int., 195(1-3):167.e1-6, 2010.

Cummings PC, Trelka DP, Kimberley M. Springer. (2011) Atlas of forensic Histopathology. Cambridge University Press,

Ciocca Gómez, L. Odontología Médico-Legal. Aspectos Forenses, Profesionales y Sociales. Santiago, Ed. Jurídicas de Santiago, 2010.

Ferrara SD, Bajanowski T, Cecchi R, Boscolo-Berto R, Viel G. (2011) Bio-medico legal scientific research in Europe: a comprehensive bibliometric overview. Int J Legal Med; 125:393-402.

Galnares Ysern A. (2012) Bibliofilia Médico Legal. Cuad Med Forense 18(3-4):143.

Gotsens M, Marí-Dell'Olmo M, Rodríguez-Sans M, Martos D, Espelt A, Pérez G, et al (2011). Validación de la causa básica de defunción en las muertes que requieren intervención médico-legal. Rev Esp Salud Pública; 85:165-76. 
Hinchliffe, J. Forensic odontology, part 2. Major disasters. Br. Dent. J., 210(6):269-74, 2011.

ICRC - International Committee of the Red Cross. Missing people, DNA analysis and identification of human remains. 2 Ed. Geneva, ICRC, 2009

INTERPOL. Guía para la Identificación de Víctimas de Catástrofes. 2009. Disponible en: http://www.interpol.int/content/download/10461/74528/version/4/file/guide[1].pdf

Krompecher, T.; Brandt-Casadevall, C.; Horisberger, B.; Perrier, M. \& Zollinger, U. The challenge of identification]

Lau G, Hui Lai S. (2008) Forensic Histopathology. En Tsokos M. Forensic Pathology Reviews. Vol. 5. Ed. Humana Press. Berlin, pp: 239-65.

Martin-de-Las-Heras, S.; Valenzuela, A.; Luna, Jde D. \& Bravo, M. The utility of dental patterns in forensic dentistry. Forensic Sci. Int., 195(1-3):166.e1-5, 2010.

McLemore, J.; Hodges, W. \& Wyman, A. Impact of identity theft on methods of identification. Am. J. Forensic Med. Pathol., 32(2):143-5, 2011.

Page, M.; Taylor, J. \& Blenkin, M. Uniqueness in the forensic identification sciences--factor fiction? Forensic Sci. Int., 206(1-3): 12-8, 2011.

Saks, M. J. Forensic identification: From a faith-based "Science" to a scientific science. Forensic Sci. Int., 201(1-3):14-7, 2010.

Varkkola, O. Dental identifications and aspects of medico-legal investigations of the Finnis Victims of the Sumatra-Andaman Earthquake on 26 December 2004. Academic Dissertation, Hjelt Institute, Department of Forensic Medicine, University of Helsinki, Finland, 2011. 